\title{
Pricing Credit Default Swaps under Lévy Models
}

\author{
Jessica Cariboni*and Wim Schoutens ${ }^{\dagger}$
}

November 22, 2004

\begin{abstract}
Most structural models for credit pricing assume Geometric Brownian motion to describe the firm asset value. However, the underlying lognormal distribution does not match empirical distributions, typically skewed and leptokurtic. Moreover, defaults are usually driven by shocks, which are not captured by the continuous paths of Brownian motion.

We assume the asset price process is driven by a pure-jump Lévy process and default is triggered by the crossing of a preset barrier. Our model incorporates asymmetry, fat-tail behaviour, jumps and instantaneous defaults. Under this model we price Credit Default Swaps, detailing the calculations for the Variance Gamma process.

Key words: Lévy process, survival probability, credit risk, structural model, credit default swap, barrier option.
\end{abstract}

AMS Subject Classifications: 60J75, 65C30

*European Commission, Joint Research Centre, I-21020 Ispra, Italy and K.U.Leuven, U.C.S., W. De Croylaan 54, B-3001 Leuven, Belgium. E-mail: Jessica.Cariboni@jrc.it

${ }^{\dagger}$ K.U.Leuven, U.C.S., W. De Croylaan 54, B-3001 Leuven, Belgium. E-mail: Wim.Schoutens@wis.kuleuven.ac.be 


\section{Introduction}

There has been an exponential growth in credit derivatives in recent years. Under the new Basel II framework banks are given the possibility to develop in-house estimates for their exposure to credit risk. Moreover, there has been renewed interest in linking credit risk with the equity market. The original idea was presented by Merton [18] (and already suggested by Black and Scholes [2]) and by Black and Cox [3]; they observed that both equities and debts can be viewed as options on the value of a firm's asset. In Merton's model default is triggered by the limited liability of the shareholders. When the value of equity drops to zero, shareholders walk away and the firm defaults on its debt. In contrast, Black and Cox's model default by means of an exogenous default barrier. The rationale behind this second approach is the fact that quite often bond covenants trigger default before the value of the equity has dropped to zero. With the Merton approach corporate bonds are modelled as American style derivatives; in the Black and Cox approach corporate bonds are barrier style products. As a consequences of all this, equity pricing techniques can be used in assessing credit risk. This simple link has been used by many practitioners over the years and numerous extensions and modifications of both types of models have been developed. A well accepted model is the CreditGrades ${ }^{\mathrm{TM}}$ model [5]. This model generalizes the standard models by monitoring default through time and uses the Geometric Brownian motion (Black-Scholes model) to describe the stock price behaviour over time. Default occurs when the stock price hits a predetermined low barrier. In order to allow for instantaneous default the default barrier is made stochastic. This leads to higher short-term spreads than those produced without the barrier uncertainty.

However, empirical evidence suggests that the underlying (log)normal distribution of this model is not accurately describing the true behaviour of the asset. Typically, the underlying distribution of stock returns is skewed and has a positive excess kurtosis. An overview of the shortcomings of the Black-Scholes model can be found for example in [22, Chapter 3]. Moreover, default events are triggered by sudden shocks in the asset price which cannot be captured by models with continuous asset path value, as the above mentioned ones.

We propose a new model under which we assume that the asset price process is described by an exponential of a (non-Brownian) Lévy process and is completely driven by jumps. Lévy based models have already proven their capabilities in equity models (see e.g. [22]) and fixed income models (see e.g. [7]). In addition, the underlying distributions in the Lévy models are very flexible and can take into account asymmetry and fat-tail behaviour. Another advantage by following this approach is that the presence of jumps in the underlying process allows for instantaneous default. Hence, there is no need to build this in artificially by making the default barrier stochastic. In conclusion, our model incorporates jumps, instantaneous default, asymmetry and fat-tail behaviour.

Other models where one is incorporating jumps in the dynamics of the firm value, are by Zhou ([24] and [25]) and by Hilberink \& Rogers [8]. While Zhou extends Longstaff and Schwartz's model [13] considering a lognormally distributed 
jump component, Hilberink \& Rogers opt for an extension of Leland's model [12] using Lévy processes which only allow for downward jumps in the firm's value.

We first formulate the model under a general Lévy market setting. Next, we show how to calculate the probability of no-default by relating it to the price of a binary barrier option. To price this option one could do a time-consuming Monte-Carlo simulation; an alternative is based on the solution of a PartialDifferential Integral Equation (PDIE). This technique has been introduced by Hirsa and Madan [9] to price American options, but can be readily adapted to price different types of barrier options. We give the details for the popular Variance Gamma (VG) model.

The model abilities are illustrated by the pricing of the most popular credit derivative: the Credit Default Swaps (CDS's). CDS's are instruments that provide the buyer an insurance against the defaulting of a company (the reference entity) on its debt. The buyer of this protection makes (continuous) predetermined payments to the seller. The payments continue until the maturity date of the contract or until default occurs, whichever is earlier. If default occurs, the buyer delivers a bond on the underlying defaulting asset in exchange for its face value. The market for CDS's is well established and trading is increasing in related products like forwards and options on these CDS's. For pricing techniques for these forwards and options see [11].

We price a CDS and the corresponding par credit spread, such that the expected premium payments on the CDS equate the expected loss payments. We show that our model can be easily calibrated to a series of realistic CDS terms structures. After the calibration procedure, the model could for example be used to price more complicated products for which there is no market price available.

The paper is organized as follows. In the next section, we give the theoretical background on Lévy Processes and pay special attention the popular example of the VG process. In Section 3, we present our new Lévy Default Model and relate CDS prices with the prices of binary barrier options. Next, in Section 4, we work out in detail the pricing algorithm for the VG model. The penultimate section reports on numerical experiments with the Lévy Default Model. We focus on computational issues, the sensitivity of the parameters and on calibration abilities of the model on CDS term structure curves observed in the market. The final section concludes.

\section{Lévy Processes}

\section{A Definition}

Suppose $\phi(z)$ is the characteristic function of a distribution. If for every positive integer $n, \phi(z)$ is also the $n$th power of a characteristic function, we say that the distribution is infinitely divisible. One can define for every such an infinitely divisible distribution a stochastic process, $X=\left\{X_{t}, t \geq 0\right\}$, called Lévy process, 
which starts at zero, has independent and stationary increments and such that the distribution of an increment over $[s, s+t], s, t \geq 0$, i.e. $X_{t+s}-X_{s}$, has $(\phi(z))^{t}$ as characteristic function.

The function $\psi(z)=\log \phi(z)$ is called the characteristic exponent and it satisfies the following Lévy-Khintchine formula [1]:

$$
\psi(z)=\mathrm{i} \gamma z-\frac{\varsigma^{2}}{2} z^{2}+\int_{-\infty}^{+\infty}\left(\exp (\mathrm{i} z x)-1-\mathrm{i} z x 1_{\{|x|<1\}}\right) \nu(\mathrm{d} x),
$$

where $\gamma \in \mathbb{R}, \varsigma^{2} \geq 0$ and $\nu$ is a measure on $\mathbb{R} \backslash\{0\}$ with $\int_{-\infty}^{+\infty}\left(1 \wedge x^{2}\right) \nu(\mathrm{d} x)<\infty$. We say that our infinitely divisible distribution has a triplet of Lévy characteristics $\left[\gamma, \varsigma^{2}, \nu(\mathrm{d} x)\right]$. The measure $\nu(\mathrm{d} x)$ is called the Lévy measure of $X$. From the Lévy-Khintchine formula, one sees that, in general, a Lévy process consists of three independent parts: a linear deterministic part, a Brownian part, and a pure jump part. The Lévy measure $\nu(\mathrm{d} x)$ dictates how the jumps occur. Jumps of sizes in the set $A$ occur according to a Poisson process with parameter $\int_{A} \nu(\mathrm{d} x)$. If $\varsigma^{2}=0$ and $\int_{-1}^{+1}|x| \nu(\mathrm{d} x)<\infty$ it follows from standard Lévy process theory [1] [21], that the process is of finite variation.

\section{B The Variance Gamma Process}

The characteristic function of the $\mathrm{VG}(\sigma, \nu, \theta)$ law is given by

$$
\phi_{V G}(u ; \sigma, \nu, \theta)=\left(1-\mathrm{i} u \theta \nu+\sigma^{2} \nu u^{2} / 2\right)^{-1 / \nu} .
$$

This distribution is infinitely divisible and one can define the VG-process $X^{(V G)}=$ $\left\{X_{t}^{(V G)}, t \geq 0\right\}$ as the process which starts at zero, has independent and stationary increments and where the increment $X_{s+t}^{(V G)}-X_{s}^{(V G)}$ over the time interval $[s, t+s]$ follows a $\operatorname{VG}(\sigma, \nu / t, t \theta)$ law:

$$
\begin{aligned}
E\left[\exp \left(\mathrm{i} u X_{t}^{(V G)}\right)\right] & =\phi_{V G}(u ; \sigma \sqrt{t}, \nu / t, t \theta) \\
& =\left(\phi_{V G}(u ; \sigma, \nu, \theta)\right)^{t} \\
& =\left(1-\mathrm{i} u \theta \nu+\sigma^{2} \nu u^{2} / 2\right)^{-t / \nu} .
\end{aligned}
$$

In [14], it was shown that the VG-process may also be expressed as the difference of two independent Gamma processes. This characterization allows the Lévy measure to be determined:

$$
\nu_{V G}(\mathrm{~d} x)=\left\{\begin{array}{ll}
C \exp (G x)|x|^{-1} \mathrm{~d} x & x<0 \\
C \exp (-M x) x^{-1} \mathrm{~d} x & x>0
\end{array},\right.
$$

where

$$
\begin{aligned}
C & =1 / \nu>0 \\
G & =\left(\sqrt{\frac{\theta^{2} \nu^{2}}{4}+\frac{\sigma^{2} \nu}{2}}-\frac{\theta \nu}{2}\right)^{-1}>0 \\
M & =\left(\sqrt{\frac{\theta^{2} \nu^{2}}{4}+\frac{\sigma^{2} \nu}{2}}+\frac{\theta \nu}{2}\right)^{-1}>0 .
\end{aligned}
$$


The Lévy measure has infinite mass, and hence a VG-process has infinitely many jumps in any finite time interval. Since $\int_{-\infty}^{+\infty}|x| \nu_{V G}(\mathrm{~d} x)<\infty$, a VGprocess has paths of finite variation. A VG-process has no Brownian component and its Lévy triplet is given by $\left[\gamma, 0, \nu_{V G}(\mathrm{~d} x)\right]$, where

$$
\gamma=\frac{-C(G(\exp (-M)-1)-M(\exp (-G)-1))}{M G} .
$$

With the parameterization in terms of $C, G$ and $M$, the characteristic function of $X_{1}^{(V G)}$ reads as follows:

$$
\phi_{V G}(u ; C, G, M)=\left(\frac{G M}{G M+(M-G) \mathrm{i} u+u^{2}}\right)^{C} .
$$

In this notation we will refer to the distribution by the notation $\operatorname{VG}(C, G, M)$.

When $\theta=0$ then $G=M$ and the distribution is symmetric. Negative values of $\theta$ lead to the case where $G<M$, resulting in negatively skewness. Similarly, the parameter $\nu=1 / C$ primarily controls the kurtosis:

\begin{tabular}{lcc}
\hline \hline & $\mathbf{V G}(\sigma, \nu, \theta)$ & $\mathbf{V G}(\sigma, \nu, 0)$ \\
\hline mean & $\theta$ & 0 \\
variance & $\sigma^{2}+\nu \theta^{2}$ & $\sigma^{2}$ \\
skewness & $\theta \nu\left(3 \sigma^{2}+2 \nu \theta^{2}\right) /\left(\sigma^{2}+\nu \theta^{2}\right)^{3 / 2}$ & 0 \\
kurtosis & $3\left(1+2 \nu-\nu \sigma^{4}\left(\sigma^{2}+\nu \theta^{2}\right)^{-2}\right)$ & $3(1+\nu)$ \\
\hline \hline
\end{tabular}

Table I: Relationships among the distribution moments and the parameters of the VG process $\sigma, \nu, \theta$.

The class of VG distributions was introduced by Madan and Seneta [16] in the late 1980s as a model for stock returns. There (and in [17] and [15]) the symmetric case $(\theta=0)$ was considered. In [14], the general case with skewness is treated.

\section{The VG Model}

The VG model is a particular example of a Lévy model in which the asset price dynamics is given as an exponential of a Lévy process. More preciesely, we consider a market consisting out of two assets, a risk-neutral bond following a price process $B=\left\{B_{t}=\exp (r t), t \geq 0\right\}$, where $r$ is the constant continuously compounded interest rate, and a risky asset with price process $S=\left\{S_{t}, t \geq 0\right\}$. We assume the asset pays out a continuous dividend yield of $q$.

The risk-neutral dynamics for the asset price are given by

$$
S_{t}=S_{0} \exp \left((r-q) t+X_{t}+\omega t\right),
$$

where $X=\left\{X_{t}, t \geq 0\right\}$ is a VG process. The risk-neutral drift rate for the asset is $r-q$ and thus to have $E\left[S_{t}\right]=S_{0} \exp ((r-q) t)$, we have to set

$$
\omega=\nu^{-1} \log \left(1-\frac{1}{2} \sigma^{2} \nu-\theta \nu\right) .
$$


For pricing of European options under this model see e.g. [14] or [22]. The pricing of American options (using the PDIE approach) for this model is given by Hirsa and Madan in [9].

\section{The Lévy Default Model}

The starting point of the model is the approach originally presented by Black and Cox [3], Leland [12], Longstaff and Schwartz [13] and the CreditGrades ${ }^{\text {TM }}$ model [5]. According to this approach an event of default occurs when the asset value of the firm crosses a deterministic barrier. This barrier corresponds to the recovery value of the firm's debt. These types of models are often referred to as asset models or first passage time structural models.

We describe the asset value of the firm by a stochastic process $S=\left\{S_{t}, t \geq\right.$ $0\}$. We then define the default event as the first crossing of some predetermined barrier $L$, which could be given in terms of e.g. debt-per-share, a global recovery on the debt etc.

We work under the so-called Lévy model, in the sense that we assume that the firm asset value follows an exponential of a Lévy process $X=\left\{X_{t}, t \geq 0\right\}$ :

$$
S_{t}=S_{0} \exp \left(X_{t}\right), \quad S_{0}>0 .
$$

Then for an initial value (i.e. time $t=0) S_{0}$ of the asset price, default is defined to occur when

$$
S_{t}=S_{0} \exp \left(X_{t}\right) \leq L
$$

or equivalently if

$$
X_{t} \leq \log \left(L / S_{0}\right) .
$$

Let us denote by $P(t)$ the risk-neutral probability of no-default between 0 and $t$ :

$$
\begin{aligned}
P(t) & =P_{Q}\left(X_{s}>\log \left(L / S_{0}\right), \text { for all } 0 \leq s \leq t\right) ; \\
& =P_{Q}\left(\min _{0 \leq s \leq t} X_{S}>\log \left(L / S_{0}\right)\right) ; \\
& =E_{Q}\left[1\left(\min _{0 \leq s \leq t} X_{s}>\log \left(L / S_{0}\right)\right)\right] ; \\
& =E_{Q}\left[1\left(\min _{0 \leq s \leq t} S_{s}>L\right)\right]
\end{aligned}
$$

where we used the indicator function $1(A)$, which is equal to 1 if the event $A$ is true and zero otherwise; the subindex $Q$ refers to the fact that we are working in a risk-neutral setting.

Consider a CDS with maturity $T$ and a continuous spread $c$. The price of a this CDS is then given by

$$
C D S=(1-R)\left(-\int_{0}^{T} \exp (-r s) \mathrm{d} P(s)\right)-c \int_{0}^{T} \exp (-r s) P(s) \mathrm{d} s,
$$


where $R$ is the asset specific recovery rate and $r$ is the default-free discount rate. From this, we find the par spread $c^{*}$ that makes the CDS price equal to zero:

$$
\begin{aligned}
c^{*} & =\frac{(1-R)\left(-\int_{0}^{T} \exp (-r s) \mathrm{d} P(s)\right)}{\int_{0}^{T} \exp (-r s) P(s) \mathrm{d} s} \\
& =\frac{(1-R)\left(1-\exp (-r T) P(T)-r \int_{0}^{T} \exp (-r s) P(s) \mathrm{d} s\right)}{\int_{0}^{T} \exp (-r s) P(s) \mathrm{d} s}
\end{aligned}
$$

Let us denote by

$$
B D O B(T, L)=\exp (-r T) E_{Q}\left[1\left(\min _{0 \leq s \leq T} S_{s}>L\right)\right],
$$

the price of a binary down-and-out barrier option with maturity $T$ and barrier level $L$; this option pays out a unit currency if the asset price $S$ remains above the barrier during the lifetime of the option and zero otherwise. Note that

$$
B D O B(T, L)=\exp (-r T) P(t) .
$$

We can rewrite the par spread $c^{*}$ in terms of the binary barrier prices as

$$
c^{*}=\frac{(1-R)\left(1-B D O B(T, L)-r \int_{0}^{T} B D O B(s, L) \mathrm{d} s\right)}{\int_{0}^{T} B D O B(s, L) \mathrm{d} s} .
$$

The problem of calculating the par spread thus reduces to the calculation of the pricing function of a binary down-and-out barrier for all maturities up to time $T$.

\section{Pricing of Digital Barrier Options}

\section{A Monte-Carlo Pricing}

Since a VG process can be seen as the difference of two independent Gamma processes, simulation of a VG process is easy.

First we introduce the Gamma processes. The density function of the Gamma distribution $\operatorname{Gamma}(a, b)$ with parameters $a>0$ and $b>0$ is given by

$$
f_{\text {Gamma }}(x ; a, b)=\frac{b^{a}}{\Gamma(a)} x^{a-1} \exp (-x b), \quad x>0 ;
$$

the corresponding characteristic function is given by

$$
\phi_{\text {Gamma }}(u ; a, b)=(1-\mathrm{i} u / b)^{-a} .
$$

Clearly, this characteristic function is infinitely divisible. The Gamma process

$X^{(\text {Gamma })}=\left\{X_{t}^{(\text {Gamma })}, t \geq 0\right\}$ with parameters $a, b>0$ is defined as the 
stochastic process which starts at zero and has stationary, independent Gamma distributed increments. More precisely, the time enters in the first parameter: $X_{t}^{(G a m m a)}$ follows a $\operatorname{Gamma}(a t, b)$ distribution.

It is easy to simulate a sample path of a Gamma process $G=\left\{G_{t}, t \geq 0\right\}$, where $G_{t}$ follows a $\operatorname{Gamma}(a t, b)$ law. We simulate the value of this process at time points $\{n \Delta t, n=0,1, \ldots\}$ as follows. First we generate independent Gamma $(a \Delta t, b)$ random numbers $\left\{g_{n}, n \geq 1\right\}$ by using a gamma random numbers generator (see e.g. [6]). Then we set

$$
G_{0}=0, \quad G_{n \Delta t}=G_{(n-1) \Delta t}+g_{n}, \quad n \geq 1
$$

A VG process $X^{(V G)}=\left\{X_{t}^{(V G)}, t \geq 0\right\}$ with parameters $C, G, M>0$ can be decomposed as $X_{t}^{(V G)}=G_{t}^{(1)}-G_{t}^{(2)}$, where $G^{(1)}=\left\{G_{t}^{(1)}, t \geq 0\right\}$ is a Gamma process with parameters $a=C$ and $b=M$ and $G^{(2)}=\left\{G_{t}^{(2)}, t \geq 0\right\}$ is a Gamma process with parameters $a=C$ and $b=G$.

In Figure 1, one sees a path of a VG process with parameters $C=20, G=40$ and $M=50$.

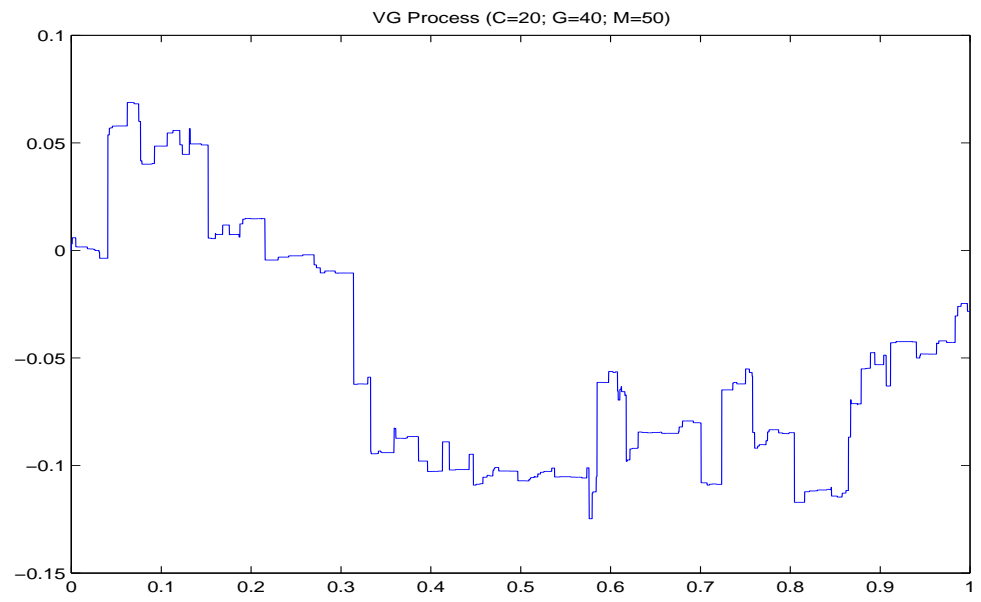

Figure 1: A sample path of a VG process

\section{B PDIE Pricing of Binary Options and the Binary Down and in Barrier Options}

First, we discuss how to price a European binary option. Let us consider a claim with maturity $T$ and payoff function 1 if $S_{T}<K$ and zero otherwise; then the price of the claim at time $t<T$ is given by

$$
V\left(S_{t}, t\right)=\exp (-r t) E_{Q}\left[1\left(S_{T}<K\right) \mid \mathcal{F}_{t}\right]
$$


where $\mathcal{F}_{t}$ is the filtration generated by the process $S_{t}$. We follow the technique introduced in [9].

It may be shown that the discounted price of the option is a martingale and hence the infinitesimal generator $\mathcal{L}$, of the underlying Lévy process (Markov process) applied to this discounted price yields zero:

$$
\mathcal{L}\left(\exp (-r t) V\left(S_{t}, t\right)\right)=0 .
$$

In our case this comes down to:

$$
\begin{gathered}
\int_{-\infty}^{+\infty}\left[V\left(S_{t-} \mathrm{e}^{x}, t\right)-V\left(S_{t-}, t\right)-\frac{\partial V}{\partial S}\left(S_{t-}, t\right) S_{t-}\left(\mathrm{e}^{x}-1\right)\right] k(\mathrm{~d} x) \\
+\frac{\partial V}{\partial t}\left(S_{t}, t\right)+(r-q) S_{t} \frac{\partial V}{\partial S}\left(S_{t}, t\right)-r V\left(S_{t}, t\right)=0,
\end{gathered}
$$

where $k(\mathrm{~d} x)$ is the Lévy measure of the underlying Lévy process.

By making the change of variables $x=\log (S)$ and $\tau=T-t$ we obtain, noting that $w(x, \tau)=V(S, t)$, the following PDIE, as a function of $w(x, \tau)$,

$$
\begin{gathered}
\int_{-\infty}^{+\infty}\left[w(x+y, \tau)-w(x, \tau)-\frac{\partial w}{\partial x}(x, \tau)\left(\mathrm{e}^{y}-1\right)\right] k(\mathrm{~d} y) \\
-\frac{\partial w}{\partial \tau}(x, \tau)+(r-q) \frac{\partial w}{\partial x}(x, \tau)-r w(x, \tau)=0
\end{gathered}
$$

This PDIE must be solved subject to the initial condition $w(x, 0)=1$ if $\mathrm{e}^{x}<K$ and zero otherwise and boundary conditions

$$
\begin{aligned}
& \frac{\partial^{2} w}{\partial x^{2}}(-\infty, \tau)-\frac{\partial w}{\partial x}(-\infty, \tau)=0, \quad \forall \tau \\
& \frac{\partial^{2} w}{\partial x^{2}}(+\infty, \tau)-\frac{\partial w}{\partial x}(+\infty, \tau)=0, \quad \forall \tau
\end{aligned}
$$

Noting that $-\omega=\int_{-\infty}^{+\infty}\left(\mathrm{e}^{y}-1\right) k(\mathrm{~d} y)$, the PDIE can be rewritten as

$$
\begin{gathered}
\int_{-\infty}^{+\infty}[w(x+y, \tau)-w(x, \tau)] k(\mathrm{~d} y) \\
-\frac{\partial w}{\partial \tau}(x, \tau)+(r-q+\omega) \frac{\partial w}{\partial x}(x, \tau)-r w(x, \tau)=0 .
\end{gathered}
$$

In Appendix 1 we work out a numerical solution scheme following [9] for this PDIE under the VG model.

To estimate the price of a binary down-and-out barrier option we first calculate the price of a binary down-and-in barrier option with barrier $L$ and time to maturity $T$. This claim pays out a unit currency if the asset price $\mathrm{S}$ goes below the barrier during the lifetime of the option and zero otherwise:

$$
B D I B(T, L)=\exp (-r T) E_{Q}\left[1\left(\min _{0 \leq s \leq T} S_{s}<L\right)\right] .
$$


To price this instrument we proceed along the same lines as done for the European binary option. The only difference is that at each time step, after computing the new values $w_{i, j+1}$ (by solving the above linear system), we impose:

$$
w_{i, j+1}=\exp (-(j+1) r \Delta t) \text { if } \mathrm{e}^{x_{i}}<L .
$$

By this we force that if the barrier $L$ has been crossed, the option always will payout one at maturity. To obtain the initial price of the binary down-and-out barrier option with barrier $L$ and time to maturity $T, B D O B(T, L)$, we can make use of the following relationship:

$$
B D I B(T, L)+B D O B(T, L)=\exp (-r T) .
$$

\section{Numerical Experiment}

\section{A Comparison of CPU Times}

We price a CDS and a binary down-and-in barrier option with time to maturity of $T=1$ year. The initial asset price is set to $S_{0}=100$, the barrier, $L$, is set equal to $50, r=0.0421$, and $q=0$. The VG-parameters used are:

$$
\sigma=0.20722, \quad \nu=0.50215, \quad \theta=-0.22898 .
$$

\begin{tabular}{lrrrrr}
\hline \hline Model & $M$ & $N$ & $c^{*}$ (in bp) & BDIB & cpu (in sec) \\
\hline PDIE VG & 50 & 50 & 121 & 0.0231 & 0.25 \\
PDIE VG & 100 & 100 & 129 & 0.0245 & 0.69 \\
PDIE VG & 150 & 150 & 131 & 0.0249 & 1.56 \\
PDIE VG & 200 & 200 & 132 & 0.0251 & 2.94 \\
PDIE VG & 250 & 250 & 132 & 0.0252 & 4.91 \\
PDIE VG & 500 & 250 & 132 & 0.0253 & 9.59 \\
PDIE VG & 250 & 500 & 132 & 0.0253 & 13.37 \\
\hline \hline Model & iterations & $N$ & $c^{*}$ (in bp) & BDIB & $c p u$ (in sec) \\
\hline MC VG & 10000 & 80 & 135 & 0.0257 & 69 \\
MC VG & 10000 & 250 & 122 & 0.0233 & 268 \\
MC VG & 100000 & 250 & 132 & 0.0252 & 2198 \\
MC VG & 500000 & 250 & 132 & 0.0251 & 11040 \\
MC VG & 1000000 & 250 & 132 & 0.0253 & 22059 \\
\hline \hline
\end{tabular}

Table II: $c^{*}$ and BDIB prices.

In the Monte-Carlo approach, the VG process is simulated as a difference of independent gamma processes. In the PDIE-approach $N$ denotes the number of time steps taken (per year); $M$ denotes the number of points in the log-strike dimension. For the CDS we assume a recovery rate $R=0.5$ and the default barrier is set to the same level $L=50$. Table II overviews different choices of 
$M, N$, and the number of iterations. Taking the Monte-Carlo price obtained by a half million iterations as a very good proxy of the true price, we observe that, in the PDIE algorithm, meshes sizes greater than $N=200$ and $M=200$, give very accurate results in quite acceptable cpu times. The Monte-Carlo approach is clearly more time consuming.

\section{B Sensitivity of the Parameters}

In the figures, we look at the sensitivity of the parameters. We first vary the kurtosis parameter $\nu$ keeping all other parameters fixed (see Figure 2). Next, we vary the skewness parameter $\theta$ and keep all other parameters fixed (see Figure 4). The results are completely inline with our intuition. Higher kurtosis (i.e. higher $\nu$ 's) give rise to higher default probabilities and higher par spreads (see Figure 3 top right graph). Also, more negative skewness (i.e. smaller $\theta$ 's) give the same effect (see Figure 5, bottom right graph).

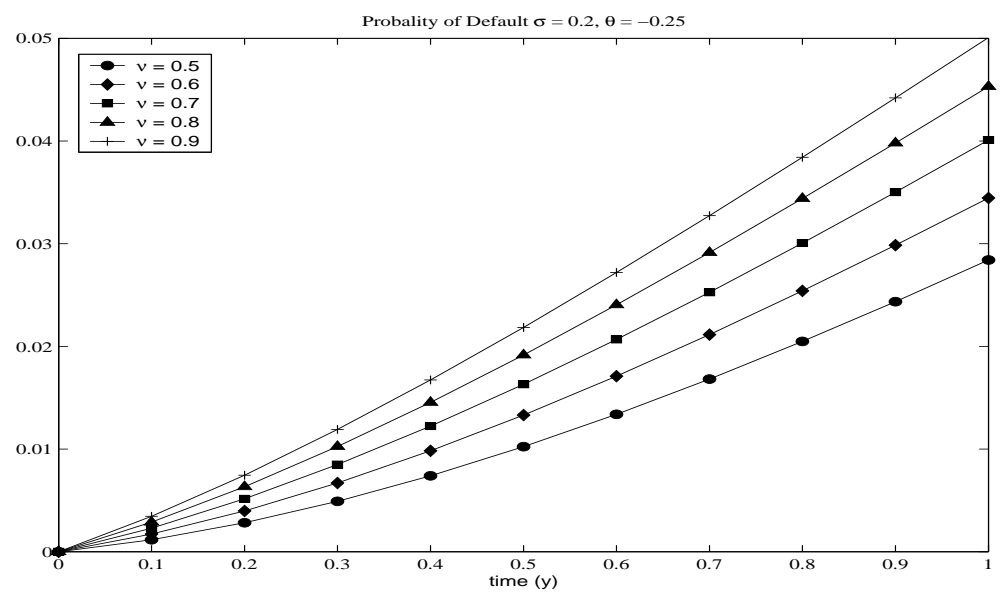

Figure 2: Kurtosis sensitivity: default probabilities. 


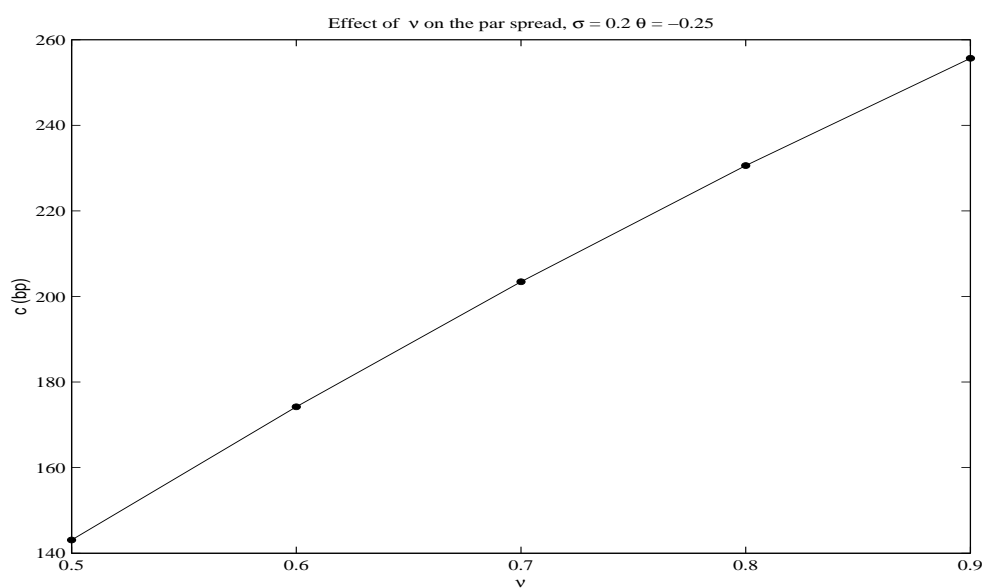

Figure 3: Kurtosis sensitivity: cds spreads 


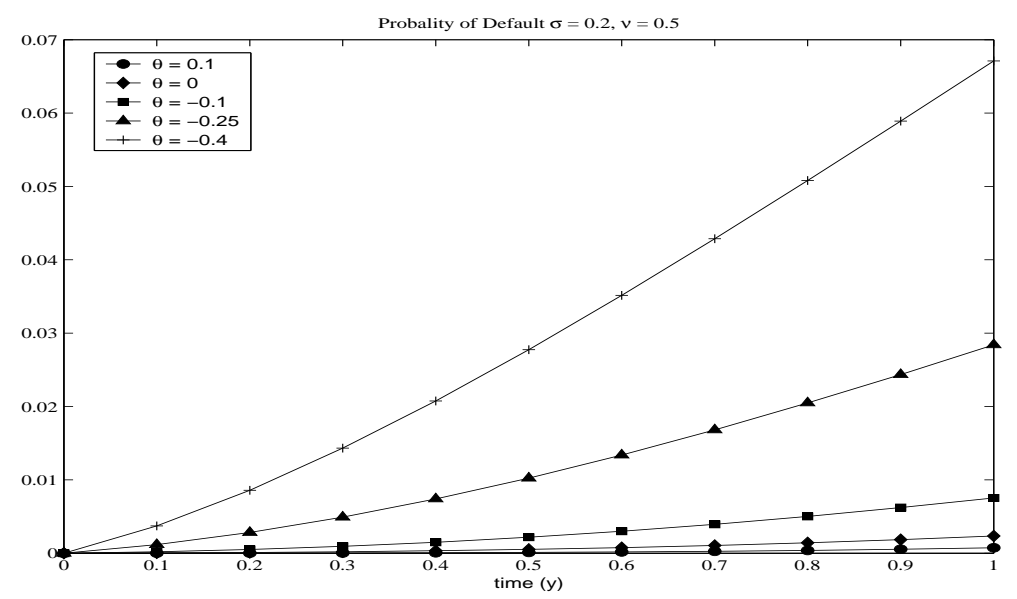

Figure 4: Skewness sensitivity: default probabilities

\section{Calibration on CDS Term Structure Curve}

Next, we calibrate the model to a series of CDS term structures which were taken from the market situation on the 26th of October 2004 and this for a whole range of differently rated (e.g. the Moody's rating) companies. We use the NelderMead simplex (direct search) method to minimize the difference between market CDS prices and calibrate the model in the least-squares sense: we minimizing the root mean square error ( $r m s e)$ given by

$$
r m s e=\sqrt{\sum_{\text {options }} \frac{(\text { Market price }- \text { Model price })^{2}}{\text { number of options }}} .
$$

In Tables III and IV we report on this calibration procedure. In the former, model prices are compared with market prices. In the latter, the optimal VGparameters are given. For comparative purposes, we also compute the average absolute error as a percentage of the mean price. This statistic, which we will denote with ape, is an overall measure of the quality of fit:

$$
\text { ape }=\frac{1}{\text { mean option price }} \sum_{\text {options }} \frac{\mid \text { Market price }- \text { Model price } \mid}{\text { number of options }}
$$

In Figure 6 and 7 the calibration for Ford and Autozone is visualized. For all the other companies, fits of the same quality were obtained. One sees clearly, that the model's CDS term structure curve captures nicely the market quotes (o-signs). 


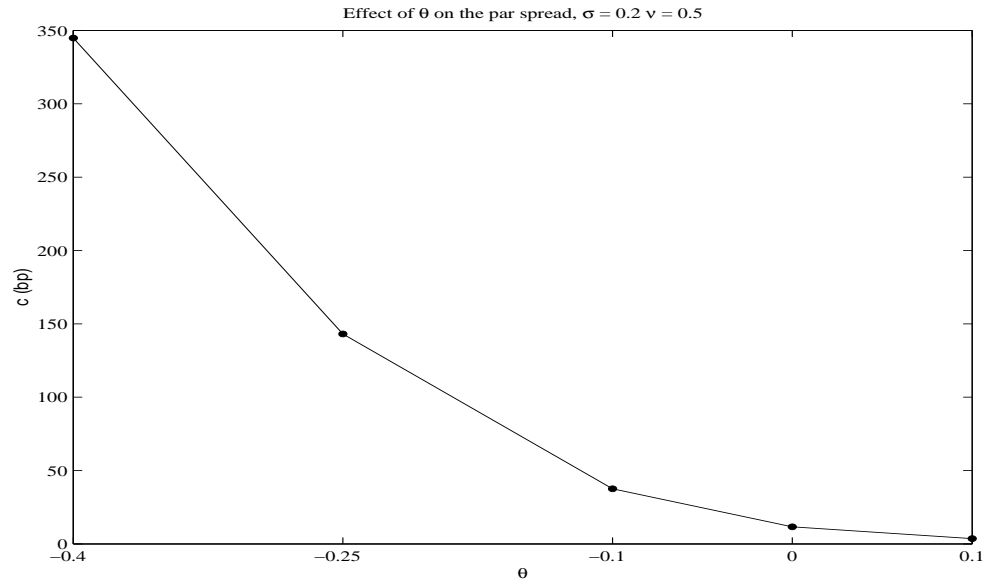

Figure 5: Skewness sensitivity: default probabilities

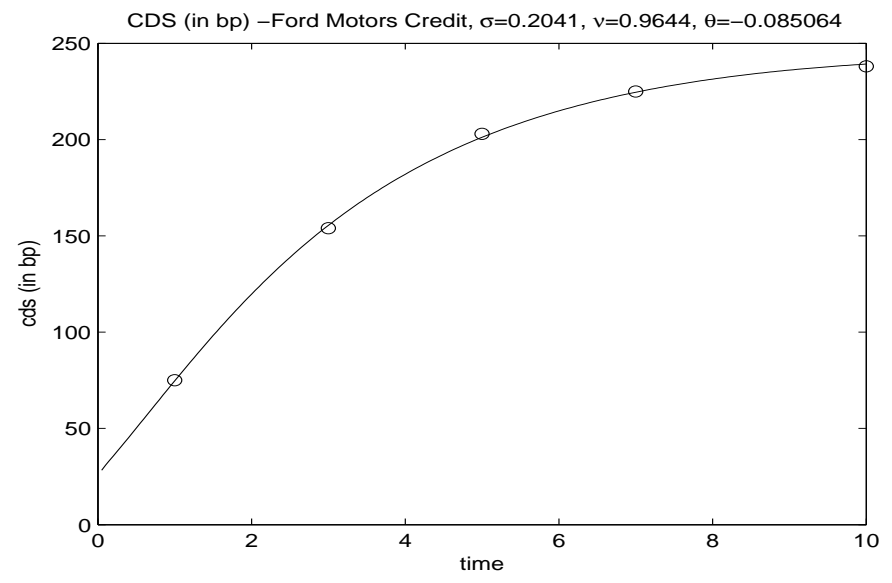

Figure 6: Calibration for Ford. 


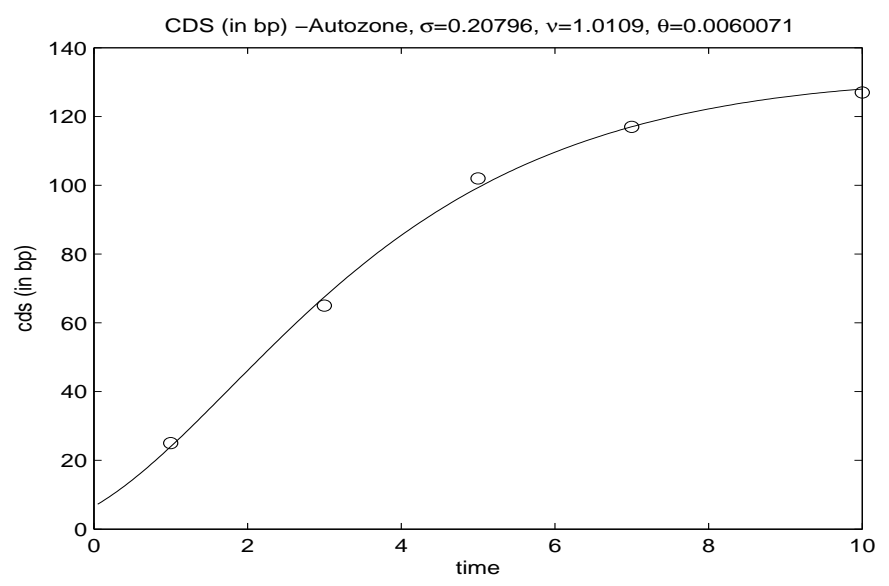

Figure 7: Calibration for Autozone 


\begin{tabular}{|c|c|c|c|c|c|c|c|}
\hline$\overline{\overline{\text { Company }}}$ & Moody & & $\overline{1 y}$ & $3 y$ & $5 y$ & $7 y$ & $\overline{10 y}$ \\
\hline Mbna Insurance & Aaa & Market & 21 & 36 & 46 & 51 & 61 \\
\hline Mbna Insurance & Aaa & Market & 21 & 35 & 46 & 53 & 60 \\
\hline General Elec. & Aaa & Market & 5 & 14 & 25 & 29 & 36 \\
\hline General Elec. & Aaa & Model & 7 & 14 & 22 & 29 & 37 \\
\hline Wells Fargo & Aa1 & Market & 3 & 10 & 20 & 23 & 32 \\
\hline Wells Fargo & Aa1 & Market & 5 & 10 & 17 & 24 & 33 \\
\hline Citigroup & Aa1 & Market & 5 & 12 & 22 & 25 & 34 \\
\hline Citigroup & Aa1 & Market & 7 & 12 & 19 & 25 & 35 \\
\hline Wal-Mart & $\mathrm{Aa} 2$ & Market & 1 & 9 & 17 & 22 & 32 \\
\hline Wal-Mart & $\mathrm{Aa} 2$ & Market & 1 & 8 & 17 & 24 & 31 \\
\hline Merrill Lynch & Aa3 & Market & 11 & 20 & 31 & 36 & 47 \\
\hline Merrill Lynch & $\mathrm{Aa} 3$ & Market & 11 & 20 & 30 & 37 & 47 \\
\hline Du Pont & Aa3 & Market & 3 & 7 & 13 & 18 & 23 \\
\hline Du Pont & Aa3 & Market & 4 & 8 & 12 & 17 & 24 \\
\hline American Express & A1 & Market & 2 & 12 & 22 & 26 & 36 \\
\hline American Express & $\mathrm{A} 1$ & Model & 3 & 12 & 21 & 28 & 35 \\
\hline Allstate & $\mathrm{A} 1$ & Market & 12 & 22 & 32 & 37 & 47 \\
\hline Allstate & A1 & Market & 12 & 22 & 31 & 38 & 47 \\
\hline Amgen & $\mathrm{A} 2$ & Market & 14 & 20 & 29 & 34 & 39 \\
\hline Amgen & $\mathrm{A} 2$ & Model & 13 & 21 & 28 & 34 & 39 \\
\hline Mcdonald's & $\mathrm{A} 2$ & Market & 3 & 10 & 19 & 23 & 34 \\
\hline Mcdonald's & $\mathrm{A} 2$ & Market & 3 & 10 & 18 & 25 & 33 \\
\hline Ford Credit Co. & A3 & Market & 75 & 154 & 203 & 225 & 238 \\
\hline Ford Credit Co. & A3 & Model & 75 & 155 & 201 & 225 & 239 \\
\hline General Motors & A3 & Market & 86 & 157 & 207 & 229 & 242 \\
\hline General Motors & A3 & Model & 90 & 156 & 199 & 227 & 252 \\
\hline$\overline{\text { Kraft Foods }}$ & A3 & Market & 4 & 19 & 31 & 40 & 51 \\
\hline Kraft Foods & $\mathrm{A} 3$ & Model & 6 & 18 & 30 & 40 & 52 \\
\hline Wyeth & Baa1 & Market & 15 & 47 & 75 & 85 & 95 \\
\hline Wyeth & Baa1 & Market & 18 & 47 & 70 & 85 & 99 \\
\hline Norfolk South. & Baa1 & Market & 3 & 12 & 28 & 34 & 44 \\
\hline Norfolk South. & Baa1 & Market & 3 & 14 & 26 & 35 & 44 \\
\hline Whirlpool & Baa1 & Market & 16 & 36 & 66 & 73 & 86 \\
\hline Whirlpool & Baa1 & Market & 17 & 39 & 59 & 74 & 89 \\
\hline Walt Disney & Baa2 & Market & 6 & 21 & 36 & 45 & 56 \\
\hline Walt Disney & Baa2 & Model & 6 & 21 & 35 & 46 & 56 \\
\hline Autozone & Baa2 & Market & 25 & 65 & 102 & 117 & 127 \\
\hline Autozone & Baa2 & Model & 24 & 67 & 99 & 117 & 127 \\
\hline Eastman Kodak & Baa3 & Market & 54 & 86 & 127 & 143 & 157 \\
\hline Eastman Kodak & Baa3 & Model & 51 & 92 & 122 & 142 & 159 \\
\hline Bombardier & Baa3 & Market & 320 & 405 & 425 & 425 & 425 \\
\hline Bombardier & Baa3 & Model & 322 & 398 & 426 & 432 & 422 \\
\hline
\end{tabular}

Table III: Calibration on CDS (in bp) term structure. Source: Goldman Sachs. 


\begin{tabular}{lrrrrr}
\hline \hline Company & $\sigma$ & $\nu$ & $\theta$ & rmse & ape \\
\hline Mbna Insurance & 0.1141 & 2.2507 & -0.0517 & 2.3354 & $2.10 \%$ \\
General Elec. & 0.0105 & 1.6186 & -0.0838 & 3.4391 & $5.62 \%$ \\
Wells Fargo & 0.0182 & 2.2513 & -0.0609 & 3.7621 & $7.81 \%$ \\
Citigroup & 0.0318 & 3.2252 & -0.0476 & 3.8059 & $7.00 \%$ \\
Wal-Mart & 0.0465 & 0.4199 & -0.1697 & 2.1339 & $5.08 \%$ \\
Merrill Lynch & 0.1446 & 2.9404 & -0.0008 & 2.1524 & $2.44 \%$ \\
Du Pont & 0.0181 & 2.8678 & -0.0484 & 2.0690 & $7.08 \%$ \\
American Express & 0.0825 & 0.5637 & -0.1221 & 2.6209 & $5.29 \%$ \\
Allstate & 0.0645 & 2.0886 & -0.0665 & 1.6894 & $1.96 \%$ \\
Amgen & 0.1528 & 3.1698 & 0.0018 & 1.8014 & $2.40 \%$ \\
Mcdonald's & 0.1043 & 1.2914 & -0.0400 & 2.3291 & $4.60 \%$ \\
Ford Credit Co. & 0.2041 & 0.9644 & -0.0851 & 2.6739 & $0.58 \%$ \\
General Motors & 0.0828 & 1.4488 & -0.1500 & 13.490 & $2.73 \%$ \\
Kraft Foods & 0.0113 & 1.0103 & -0.1168 & 2.8559 & $3.77 \%$ \\
Wyeth & 0.0111 & 0.7712 & -0.1716 & 7.2242 & $3.94 \%$ \\
Norfolk South. & 0.1009 & 0.5032 & -0.1137 & 3.1334 & $4.50 \%$ \\
Whirlpool & 0.0428 & 1.1204 & -0.1241 & 8.5203 & $5.41 \%$ \\
Walt Disney & 0.1400 & 0.8057 & -0.0453 & 1.1744 & $1.26 \%$ \\
Autozone & 0.2080 & 1.0109 & 0.0060 & 3.9249 & $1.65 \%$ \\
Eastman Kodak & 0.2123 & 2.4285 & -0.0066 & 8.0492 & $2.68 \%$ \\
Bombardier & 0.3553 & 2.8132 & -0.0824 & 10.6213 & $1.00 \%$ \\
\hline \hline
\end{tabular}

Table IV: Calibration on CDS (in bp) term structure. Source: Goldman Sachs. 


\section{Conclusions}

In this work we have introduced a new structural model to price credit derivatives. As in classical structural models, an event of credit default is assumed to occur when the asset value of a given firm crosses a preset barrier. In contrast to classical models where path-continuous Brownian motion is supposed for the asset value, we assume the asset price to follow the exponential of a pure-jump Lévy process.

Opposite to the Gaussian case, the underlying distribution in Lévy models can be asymmetric and leptokurtic, like typically observed empirical asset distributions. Moreover, the presence of jumps in the process allows for unexpected default, which is instead introduced artificially, e.g. via a stochastic barrier, in continuous path models.

Under this structural Lévy model, we have calculated the survival probability for the firm by relating it to the price of a binary down-and-out barrier option. For the Variance Gamma process we have detailed the calculations of the survival probability. In this case the estimation of the price of the barrier option can be obtained by solving a partial integral differential equation.

We have tested the capabilities of our model by pricing a credit default swap and estimating the correspondent par spread. The partial differential integral approach has allowed obtaining accurate results in acceptable cpu times. Specifically, the gain in cpu time with respect to a Monte-Carlo approach is of the order of 1000 . We have verified that, as expected, fat-tails or negative asymmetries in the underlying distribution yields higher default probabilities. Finally the model has been calibrated to a series of market credit default structures. Results of this calibration show that the model we propose can adequately match typical features of observed structure curves. 


\section{Appendix 1: Solution of the PDIE}

In the finite difference discretization of equation (27), a mixed approach is used. For the evaluation of the jumps term, the integrand is expanded near its singularity at zero and this part is treated implicitly. The rest of the integral is instead treated explicitly, in order for the scheme to be computationally affordable. On the rest of the PDIE, a fully implicit approach is used.

We consider $M$ equally spaced sub-intervals in the $\tau$-direction. For the $x$ direction we assume $N$ equally spaced sub-intervals on $\left[x_{\min }, x_{\max }\right]$. Denoting by

$$
\Delta x=\left(x_{\max }-x_{\min }\right) / N \text { and } \Delta \tau=T / M,
$$

this leads to the following mesh on $\left[x_{\min }, x_{\max }\right] \times[0, T]$ :

$D=\left\{\left(x_{i}, \tau_{j}\right) \in \mathbb{R}^{+} \times \mathbb{R}^{+} \mid x_{i}=x_{\min }+i \Delta x, i=0,1, \ldots, N ; \tau_{j}=j \Delta \tau, j=0,1, \ldots, M\right\}$.

Let $w_{i, j}$ be the discrete value of $w\left(x_{i}, \tau_{j}\right)$ on $D$. Using the first order finite difference approximation for $\partial w / \partial \tau$ and central difference for $\partial w / \partial x$, we obtain the following discrete equation at point $\left(x_{i}, \tau_{j+1}\right)$ :

$$
\begin{array}{r}
\int_{-\infty}^{+\infty}\left[w\left(x_{i}+y, \tau_{j}\right)-w\left(x_{i}, \tau_{j}\right)\right] k(\mathrm{~d} y)-\frac{1}{\Delta \tau}\left(w_{i, j+1}-w_{i, j}\right) \\
\quad+(r-q+\omega) \frac{1}{2 \Delta x}\left(w_{i+1, j+1}-w_{i-1, j+1}\right)-r w_{i, j+1}=0
\end{array}
$$

Equivalently,

$$
\begin{gathered}
(r-q+\omega) \frac{\Delta \tau}{2 \Delta x} w_{i-1, j+1}-(r-q+\omega) \frac{\Delta \tau}{2 \Delta x} w_{i+1, j+1}+(1+r \Delta \tau) w_{i, j+1}, \\
=w_{i, j}+\Delta \tau \int_{-\infty}^{+\infty}\left[w\left(x_{i}+y, \tau_{j}\right)-w\left(x_{i}, \tau_{j}\right)\right] k(\mathrm{~d} y)
\end{gathered}
$$

where $w_{i, 0}=1$ if $e^{x_{i}}<K$ and zero otherwise.

For the evaluation of the jump integral we use an analytical approach to the singularity at zero combined with an explicit approach. We divide it to six 
integrals, resp. given by $A_{1}, A_{2}, A_{3}, A_{4}, A_{5}$ and $A_{6}$ :

$$
\begin{aligned}
\int_{-\infty}^{+\infty}\left[w\left(x_{i}+y, \tau_{j}\right)-w\left(x_{i}, \tau_{j}\right)\right] k(\mathrm{~d} y) & =\int_{-\infty}^{x_{\min }-x_{i}}\left[w\left(x_{i}+y, \tau_{j}\right)-w\left(x_{i}, \tau_{j}\right)\right] k(\mathrm{~d} y) \\
& +\int_{x_{\min }-x_{i}}^{-\Delta x}\left[w\left(x_{i}+y, \tau_{j}\right)-w\left(x_{i}, \tau_{j}\right)\right] k(\mathrm{~d} y) \\
& +\int_{-\Delta x}^{0}\left[w\left(x_{i}+y, \tau_{j}\right)-w\left(x_{i}, \tau_{j}\right)\right] k(\mathrm{~d} y) \\
& +\int_{0}^{\Delta x}\left[w\left(x_{i}+y, \tau_{j}\right)-w\left(x_{i}, \tau_{j}\right)\right] k(\mathrm{~d} y) \\
& +\int_{\Delta x}^{x_{\max }-x_{i}}\left[w\left(x_{i}+y, \tau_{j}\right)-w\left(x_{i}, \tau_{j}\right)\right] k(\mathrm{~d} y) \\
& +\int_{x_{\max }-x_{i}}^{\infty}\left[w\left(x_{i}+y, \tau_{j}\right)-w\left(x_{i}, \tau_{j}\right)\right] k(\mathrm{~d} y) \\
& =A_{1}+A_{2}+A_{3}+A_{4}+A_{5}+A_{6}
\end{aligned}
$$

Following [9], one has

$$
\begin{aligned}
A_{1} \cong & \nu^{-1}\left(\mathrm{e}^{-r t_{j}}-w_{i, j}\right) \operatorname{expint}\left(i \Delta x \lambda_{n}\right) \\
A_{2} \cong & \sum_{k=1}^{i-1} \nu^{-1}\left[w_{i-k, j}-w_{i, j}-k\left(w_{i-k-1, j}-w_{i-k, j}\right)\right]\left[\operatorname{expint}\left(k \Delta x \lambda_{n}\right)-\operatorname{expint}\left((k+1) \Delta x \lambda_{n}\right)\right] \\
& \quad+\sum_{k=1}^{i-1}\left(\lambda_{n} \nu \Delta x\right)^{-1}\left(w_{i-k-1, j}-w_{i-k, j}\right)\left(\mathrm{e}^{-\lambda_{n} k \Delta x}-\mathrm{e}^{-\lambda_{n}(k+1) \Delta x}\right) \\
A_{3} \cong & \left(\nu \Delta x \lambda_{n}\right)^{-1}\left(1-\mathrm{e}^{-\lambda_{n} \Delta x}\right)\left(w_{i-1, j}-w_{i, j}\right) \\
A_{4} \cong & \left(\nu \Delta x \lambda_{p}\right)^{-1}\left(1-\mathrm{e}^{-\lambda_{p} \Delta x}\right)\left(w_{i+1, j}-w_{i, j}\right) \\
& \quad \sum_{k=1}^{N-i-1} \nu^{-1}\left[w_{i+k, j}-w_{i, j}-k\left(w_{i+k+1, j}-w_{i+k, j}\right)\right]\left[\operatorname{expint}\left(k \Delta x \lambda_{p}\right)-\operatorname{expint}\left((k+1) \Delta x \lambda_{p}\right)\right] \\
& \quad+\sum_{k=1}^{N-i-1}\left(\lambda_{p} \nu \Delta x\right)^{-1}\left(w_{i+k+1, j}-w_{i+k, j}\right)\left(\mathrm{e}^{-\lambda_{p} k \Delta x}-\mathrm{e}^{-\lambda_{p}(k+1) \Delta x}\right)
\end{aligned}
$$

$A_{6} \cong \nu^{-1} w_{i, j} \operatorname{expint}\left((N-i) \Delta x \lambda_{p}\right)$,

since for $y \in\left(-\infty, x_{\min }-x_{i}\right)$, we have $w\left(x_{i}+y, t_{j}\right) \cong \mathrm{e}^{-r t_{j}}$ and for $y \in$ $\left(x_{\max }-x_{i}, \infty\right)$, we have $w\left(x_{i}+y, t_{j}\right) \cong 0$.

Putting all pieces together, we obtain the following difference equation at the points $\left(x_{i}, \tau_{j+1}\right)$

$$
A w_{i-1, j+1}+B_{i} w_{i, j+1}-C w_{i+1, j+1}=w_{i, j}+\nu^{-1} \Delta \tau R_{i, j},
$$


where

$$
\begin{aligned}
A= & (r-q+\omega) \frac{\Delta \tau}{2 \Delta x}-\left(1-\mathrm{e}^{-\lambda_{n} \Delta x}\right) \frac{\Delta \tau}{\nu \Delta x \lambda_{n}} \\
B_{i}= & 1+r \Delta \tau+\left(1-\mathrm{e}^{-\lambda_{n} \Delta x}\right) \frac{\Delta \tau}{\nu \Delta x \lambda_{n}}+\left(1-\mathrm{e}^{-\lambda_{p} \Delta x}\right) \frac{\Delta \tau}{\nu \Delta x \lambda_{p}} \\
& +\frac{\Delta \tau}{\nu}\left(\operatorname{expint}\left(i \Delta x \lambda_{n}\right)+\operatorname{expint}\left((N-i) \Delta x \lambda_{p}\right)\right) \\
C= & (r-q+\omega) \frac{\Delta \tau}{2 \Delta x}+\left(1-\mathrm{e}^{-\lambda_{p} \Delta x}\right) \frac{\Delta \tau}{\nu \Delta x \lambda_{p}} \\
R_{i, j}= & \sum_{k=1}^{i-1}\left(w_{i-k, j}-w_{i, j}-k\left(w_{i-k-1, j}-w_{i-k, j}\right)\right)\left(\operatorname{expint}\left(k \Delta x \lambda_{n}\right)-\operatorname{expint}\left((k+1) \Delta x \lambda_{n}\right)\right) \\
& +\sum_{k=1}^{i-1}\left(\lambda_{n} \Delta x\right)^{-1}\left(w_{i-k-1, j}-w_{i-k, j}\right)\left(\mathrm{e}^{-\lambda_{n} k \Delta x}-\mathrm{e}^{-\lambda_{n}(k+1) \Delta x}\right) \\
& +\sum_{k=1}^{N-i-1}\left(w_{i+k, j}-w_{i, j}-k\left(w_{i+k+1, j}-w_{i+k, j}\right)\right)\left(\operatorname{expint}\left(k \Delta x \lambda_{p}\right)-\operatorname{expint}\left((k+1) \Delta x \lambda_{p}\right)\right) \\
& +\sum_{k=1}^{N-i-1}\left(\lambda_{p} \Delta x\right)^{-1}\left(w_{i+k+1, j}-w_{i+k, j}\right)\left(\mathrm{e}^{-\lambda_{p} k \Delta x}-\mathrm{e}^{-\lambda_{p}(k+1) \Delta x}\right) \\
& +\mathrm{e}^{-r \tau_{j}} \operatorname{expint}\left(i \Delta x \lambda_{n}\right)
\end{aligned}
$$

Assuming that at time $\tau_{j}$ we know the values $w_{i, j}$, we compute for time $\tau_{j+1}$ the values $w_{i, j+1}$ by solving the above (tri-diagonal) linear system. We always impose the boundary conditions in case of $i=1$ or $i=N$.

\section{Acknowledgments}

The second author is a Postdoctoral Fellow of the Fund for Scientific Research - Flanders (Belgium) (F.W.O. - Vlaanderen). The authors thank Geert Gielens (K.U.Leuven and Dexia Bank) for his inspiring ideas, fruitful discussion and stimulating comments and Goldman Sachs for kindly providing CDS data. 


\section{References}

[1] J. Bertoin, 1996, Lévy Processes (Cambridge Tracts in Mathematics 121, Cambridge University Press, Cambridge).

[2] F. Black, and M. Scholes, 1973, The pricing of options and corporate liabilities, Journal of Political Economy, 81, 637-654.

[3] F. Black, and J. Cox, 1976, Valuing corporate securities: some effects on bond indenture provisions, Journal of Finance, 31, 351-367.

[4] P. Carr, and D.B. Madan, 1998, Option Valuation using the Fast Fourier Transform, Journal of Computational Finance, 2, 61-73.

[5] - , 2002, CreditGrades ${ }^{\mathrm{TM}}$, Technical Document, RiskMetrics Group, Inc.

[6] L. Devroye, 1986, Non-Uniform Random Variate Generation (Springer).

[7] E. Eberlein, and S. Raible, 1999, Term structure models driven by general Lévy processes, Mathematical Finance, 9(1), 31-53.

[8] B. Hilberink and L. C. G. Rogers, 2002, Optimal Capital Structure and Endogenous Default, Finance and Stochastics, 6, 237-263.

[9] A. Hirsa, and D.B. Madan, 2003, Pricing American options under Variance Gamma. Journal of Computational Finance, $7(2)$.

[10] J.C. Hull, 2000, Options, Futures and Other Derivatives (4th edition, Prentice-Hall).

[11] J.C. Hull, and A. White, 2003, The Valuation of Credit Default Swap Options, Journal of Derivatives, 10(3), 40-50.

[12] H. Leland, 1994, Corporate debt value, bond convenants, and optimal capital structure, Journal of Finance, 49, 1213-1252.

[13] F. Longstaff, and E. Schwartz, 1995, A simple approach to valuing risky fixed and floating rate debtebt value, bond convenants, and optimal capital structure, Journal of Finance, 50, 789-819.

[14] D.B. Madan, P. Carr, and E.C. Chang, 1998, The variance gamma process and option pricing. European Finance Review, 2, 79-105.

[15] D.B. Madan, and F. Milne, 1991, Option pricing with V.G. martingale components. Mathematical Finance, 1(4), 39-55.

[16] D.B. Madan and E. Seneta, 1987, Chebyshev polynomial approximations and characteristic function estimation. Journal of the Royal Statistical Society Series B, 49(2), 163-169.

[17] D.B. Madan and E. Seneta, 1990, The v.g. model for share market returns, Journal of Business, 63, 511-524. 
[18] R. Merton, 1974, On the pricing of corporate debt: the risk structure of interest rates. Journal of Finance, 29, 449-470.

[19] G. Pan, 2001, Equity to credit pricing, Risk, November issue, 99-102.

[20] S. Raible, 2000, Lévy Processes in Finance: Theory, Numerics, and Empirical Facts, Ph.D. thesis, Freiburg i. Br..

[21] K. Sato, 2000, Lévy Processes and Infinitely Divisible Distributions (Cambridge Studies in Advanced Mathematics 68, Cambridge University Press, Cambridge).

[22] W. Schoutens, 2003, Lévy Processes in Finance: Pricing Financial Derivatives (Wiley).

[23] M. Yor, and L. Nguyen, 2001, Wiener-Hopf Factorization and the Pricing of Barrier and Lookback Options under General Lévy Processes (Prépublications du Laboratoire de Probabilités et Modèles Aléatoires 640, Universités de Paris 6 \& Paris 7, Paris).

[24] C. Zhou, 1997, A Jump-Diusion Approach to Modelling Credit Risk and Valuing Defaultable Securities (Federal Reserve Board, Washington).

[25] C. Zhou, 2001, The Term Structure of Credit Spreads with Jump Risk, Journal of Banking and Finance, 25, 2015-2040. 